\title{
Experimental determination of stiffness and damping in rotating systems using metaheuristic hybrid optimization and state observers
}

\author{
Lucas Rangel de Oliveira ${ }^{1}$ - Gilberto Pechoto de Melo ${ }^{1}$
}

Received: 24 April 2015 / Accepted: 11 August 2015 / Published online: 3 September 2015

(C) The Brazilian Society of Mechanical Sciences and Engineering 2015

\begin{abstract}
Recently, more research have been conducted on the analysis of the vibration response of rotors, so that new techniques, which are used to characterize the dynamic response of rotating machines, have been developed to aid and predict the lifespan of such systems. One of the vibration management techniques in rotating systems is the monitoring of stiffness and damping parameters of the bearings and system excitation forces. In this context, this paper aims to estimate the stiffness and damping of the bearings through a hybrid metaheuristic method. This uses a population-based search method as a starting point for direct search method, genetic algorithms and quasi-Newton method, respectively, in this work. The function to be minimized contains experimental bearing data. Moreover, the state observer is used to reconstruct all states of the system from the experimental data from the sensors. Thus, it is proposed to identify the excitation forces using orthogonal functions, Chebyshev polynomials specifically, and then, to verify the effectiveness of the method when compared to the calculated force excitation of the system.
\end{abstract}

Keywords Unbalance force - Quasi-Newton method . Genetic algorithm · State observers $\cdot$ Chebyshev polynomials

Technical Editor: Kátia Lucchesi Cavalca Dedini.

Lucas Rangel de Oliveira

lucas.unesp.oliveira@gmail.com

Gilberto Pechoto de Melo

gilberto@dem.feis.unesp.br

1 Department of Mechanical Engineering, Faculdade de Engenharia de Ilha Solteira, UNESP-Univ Estadual Paulista, Ilha Solteira, SP, Brazil

\author{
Abbreviations \\ [A] Dynamic matrix \\ $[B] \quad$ Input matrix \\ [C] Damping matrix \\ $\left[D_{s}\right] \quad$ Output matrix \\ $\left[D_{m e}\right] \quad$ Measurement matrix \\ $e \quad$ Distance between the center and the unbalanced \\ mass \\ $[F],[X]$ Force and displacement coefficient to Chebyshev \\ polynomials \\ $[G] \quad$ Gyroscopic matrix \\ [I] Identity matrix \\ $J \quad$ Cost function \\ $\left[L_{o b}\right] \quad$ Gain matrix of the observer \\ $m_{o} \quad$ Unbalanced mass \\ [M] Mass matrix \\ $[P] \quad$ Operational matrix of integration \\ $r \quad$ Number of terms of Chebyshev polynomials \\ $\delta \quad \quad$ Nodal displacement vector for an element \\ $\Omega \quad$ Angular velocity
}

\section{Introduction}

Many industries working with rotating machinery would like to have monitoring systems. Therefore, that they can work safely and abrupt stop can be avoided. Unbalance, misalignment and bearing failure are responsible for vibration, which depending on the intensity can cause damage [2].

One technique that is widespread today is the identification of unbalance forces and bearing parameters, in either the time domain or the frequency domain $[6,8]$.

Various methods have been developed for the identification of dynamic system parameters using orthogonal 
functions, in recent years, but none can be considered universally suitable for all situations $[6,8]$.

Simões and Júnior [10] in 2002 used a method based on a genetic algorithm to characterize the presence of a fault in the rotor system, by measuring the change in stiffness of the shaft caused by the presence of faults.

In 2010, Castro et al. [1] proposed a hybrid optimization method, which uses a genetic algorithm together with simulated annealing to identify the magnitude and phase of the unbalance and position of a rotating system supported by hydrodynamic bearings. The model allowed the simulation of the rotor orbits.

In 2003, Pacheco et al. [8] used five types of orthogonal functions, including Chebyshev polynomials, to simulate the input and output signals to a system with one and two degrees of freedom. Compared with other orthogonal functions, Chebyshev polynomials showed good performance, requiring the least number of terms.

In 2007, Melo [6] presented a method for identifying parameters in mechanical systems, which relies on the expansion of excitation functions and system response in terms of the Fourier series, and transformation of the differential equations of the motion to algebraic equations by successive integration, using the operational matrix integration.

However, the identification of bearing parameters using optimization methods and the excitation force identified by orthogonal function method in rotating systems is not yet widely explored.

\section{Methodology}

The finite element method was used to modeling a particular rotor where there is a division of the continuous system in a finite number of elements, which are connected by nodes. The finite element model used has four degrees of freedom per node, two displacements $v$ and $w$ and two rotations, $\theta$ and $\psi$, of the end element as shown in Fig. 1 [3].

Using standard finite element formulation, the nodal displacement vector for an element is defined by the Eq. (1).

$\delta=\left[v_{1}, w_{1}, \theta_{1}, \psi_{1}, v_{2}, w_{2}, \theta_{2}, \psi_{2}\right]^{T}$

For each shaft element, there is a finite element model that describes its mass properties, the rotational inertia of gyroscopic effect and stiffness, represented by the matrix given by the equations described by Lalanne [1].

To develop methodologies to identify the excitation force and the dynamic system parameters there is a necessity to build mathematical models to represent the mechanical behavior of various types of systems. Therefore, equation of motion is given by Eq. (2).

$[M]\{\ddot{x}(t)\}+([C]+[G])\{\dot{x}(t)\}+[K]\{x(t)\}=\{f(t)\}$

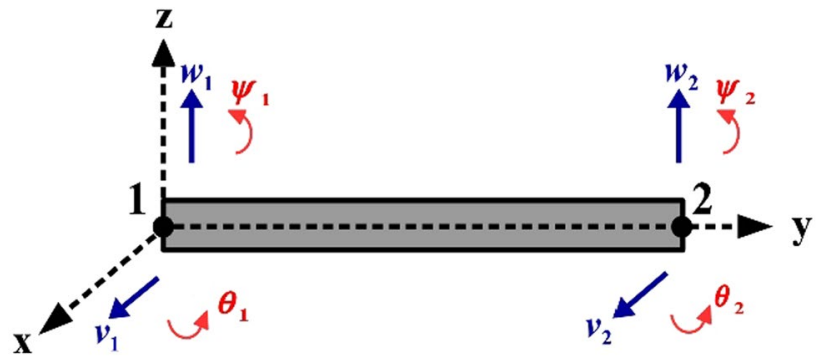

Fig. 1 Degrees of freedom of the finite element

where $\ddot{x}(t), \dot{x}(t)$ and $x(t)$, respectively, are vectors of acceleration, velocity and displacement; $[M]$ is the mass matrix; $[G]$ is the gyroscopic effect matrix; $[C]$ is damping matrix; $[K]$ is the stiffness matrix and $\{f(t)\}$ is the vector of external forces [3].

Equation (2) can be written in state space form as:

$\{\dot{x}(t)\}=[A]\{x(t)\}+[B]\{u(t)\}$

$\{y(t)\}=\left[D_{s}\right]\{x(t)\}$

where

$[A]=\left[\begin{array}{cc}{[0]_{n \times n}} & {[I]_{n \times n}} \\ -[K]_{n \times n}[M]_{n \times n}^{-1} & -[C+G]_{n \times n}[M]_{n \times n}^{-1}\end{array}\right]_{2 n \times 2 n}$
$[B]=\left[\begin{array}{c}{[0]_{n \times n}} \\ {[M]_{n \times n}^{-1}}\end{array}\right]_{2 n \times n}$

$\left[D_{s}\right]=[I]_{n \times n}$

The matrix $[A]$, composed of mass, damping, stiffness, zeroes, $[0]$, and identity, $[I]$, matrix, is called dynamic matrix, which has the function to couple the state variables, the $[B]$ matrix is called input matrix, which have the function of directing and weight of the inputs system, the matrix $\left[D_{s}\right]$ is called output matrix, which has the function of directing and weight of the state variables, $\{x(t)\}$ is the state variable vector, $\{y(t)\}$ is the output vector and $\{u(t)\}$ is the input vector [4].

However, there is difficulty in assembling the stiffness and damping matrices due to the difficulty in obtaining the values of these parameters for the modeling of bearings. It is proposed to use a hybrid optimization method, with a population-based method followed a direct search method.

The population-based methods, in particular genetic algorithms, are suitable for complex optimization problems, which involve many variables and a space of higher dimension solutions. The drawback of these algorithms is the introduction of an "overhead" computer, or an excess processing or storage causing a high computational cost due to the large number of variables, to a large population and a high number of generations to cover the space of solutions [1]. 
The genetic algorithm operates with a set of individuals (population) in which each one can make desired solution. The function $f\left(x_{1}, x_{2}, \ldots, x_{n}\right)$ to be optimized, called the objective represents the environment in which the initial population will be exposed.

This optimization function is designed to calculate the quadratic norm of root-mean-square values, rms, of the responses of the analytical model, $y(t)$, obtained by the state observer and the responses obtained experimentally through sensors, $y_{\exp }(t)$, at $n$ positions of the rotor. The analytical model, where $y_{\text {sim }}(t)$ is obtained, containing the parameters that the bearings are adjusted to minimize the function $f(x)$, selecting parameters that best represent the system.

$f\left(x_{1}, x_{2}, \ldots, x_{n}\right)=\left(\sum_{i=1}^{n}\left(y_{\exp (i)}(t)-y_{\operatorname{sim}(i)}(t)\right)^{2}\right)^{1 / 2}$

Through the mechanisms of evolution, the fittest individuals are more likely to reproduce and with each new generation, a group becomes fittest by minimizing the function to be optimized.

After the selection of the fittest is carried out, the next step is the application of genetic operators aimed at diversifying the individuals so that they are more able to the environment, among the most common are the crossover and the mutation [10].

Finally, after the selection and application of genetic operators, there is a new generation, which will be evaluated. If the present generation is not yet adapted, the process is repeated until the objective function reaches its minimum value or it reaches a stopping criterion [1, 7, 12]. The basic genetic algorithm can be summarized in the following steps, as shown in Fig. 2.

The conditions used in the genetic algorithm are shown in Table 1.

The optimization by direct search, such as quasi-Newton method, are also used to get a vector that maximizes or minimizes a particular function. However, for the start of the search is necessary to adopt an array initial solution. If the vector chosen as the starting point is near the real, the algorithm finds an optimal scheduling making minor modification this solution, seeking improvements in the results of the objective function and does not require a big time for this. The algorithm ends when there is no possible improvement in one iteration or when it meets one of the stopping criteria previously provided to the algorithm. The solution, at the end of the algorithm, is ideally great, but there is no unique guarantee.

The quasi-Newton method comprises to find a new vector solution at each iteration according to Eq. (5).

$x^{(k+1)}=x^{(k)}-\alpha^{(k)} H^{*(k)} g^{(k)}$

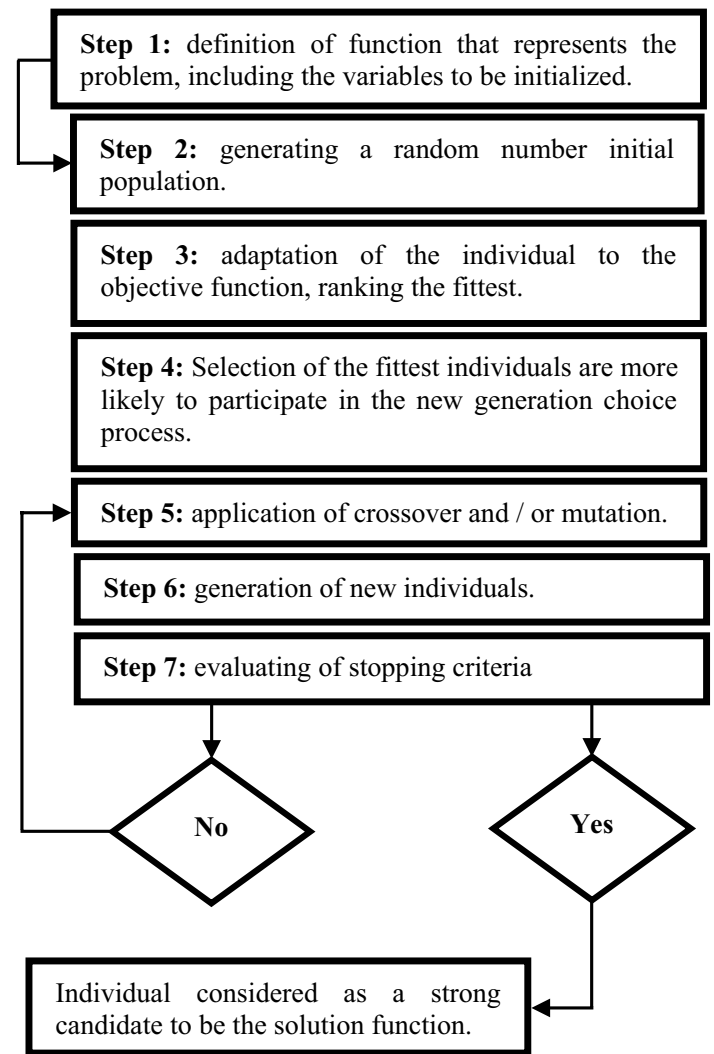

Fig. 2 General steps of a genetic algorithm

Table 1 Conditions given the genetic algorithm

\begin{tabular}{ll}
\hline Parameters & Values \\
\hline Number of variables & 8 \\
Population size & 80 \\
Initial range & -100 to 1000 \\
Selection method & Stochastic Universal Sampling \\
Mutation operator & Gaussian \\
Number of generations & 800 \\
Crossover rate & 0.8 \\
Crossover method & Arithmetic \\
Migration of Fraction & 0.2 \\
Migration Range & 20 \\
Elitism & 2 \\
Stopping criteria (fitness function) & $10^{-2}$ \\
\hline
\end{tabular}

and $\alpha^{(k)}$ is a scalar chosen so as to minimize the function, $g^{(k)}$ is the gradient of the function and $H^{*(k)}$ is the Hessian approximation $[2,5,9]$.

In view of the advantages and disadvantages of both methods, hybrid algorithms use the based population search methods as a starting point for direct search method, genetic algorithms and quasi-Newton method, respectively, in this work. 
As mentioned above, it is indisputable the importance of fully know the state vector of the dynamic system. However, many system variables may not be available for measuring or, where available, require a large number of sensors. Thus, this variables must be estimated.

Luenberger (1964) showed that the states of a deterministic dynamic system are estimated from the knowledge of their inputs and their outputs, since this system is observable, or at least detectable. This device, based on a control system closed loop, is known as Luenberger Observer [2].

Knowing $A, B$ and $D_{s}$ and the measurements of $y(t)$, and $u(t)$, it is possible to construct an observer system as follows in Eq. (4) $[4,6]$.

$$
\begin{aligned}
& \{\dot{\hat{x}}(t)\}=[A]\{\hat{x}(t)\}+[B]\{u(t)\}+\left[L_{o b}\right]\{y(t)-\hat{y}(t)\} \\
& \{\hat{y}(t)\}=\left[D_{m e}\right]\{\hat{x}(t)\}
\end{aligned}
$$

where $\{\hat{x}(t)\}$ is the estimated input signal vector, $\{\hat{y}(t)\}$ is the estimated output signal vector, $\left[D_{m e}\right]$ is the measures matrix, which indicate the parameter that is measured for the reconstruction of other states and $\left[L_{o b}\right]$ is the gain matrix of the observer. Thus, it is possible to simulate the original system with the observed states, as shown in Fig. 3.

The gain matrix of the observer, $\left[L_{o b}\right]$ tries to minimize iteratively the error between the output simulated and the output estimated by the observer, approaching the estimated state by the observer from analytical state obtained by system modeling. This is done by minimizing a cost function $[J(t, x, u)]$, defined by Eq. (7) $[6,7]$.

$[J(t, x, u)]=\int_{0}^{\infty}\left[\left\{x^{T}(\tau)\right\}[Q]\{x(\tau)\}+\left\{u^{T}(\tau)\right\}[R]\{u(\tau)\}\right] \mathrm{d} \tau$

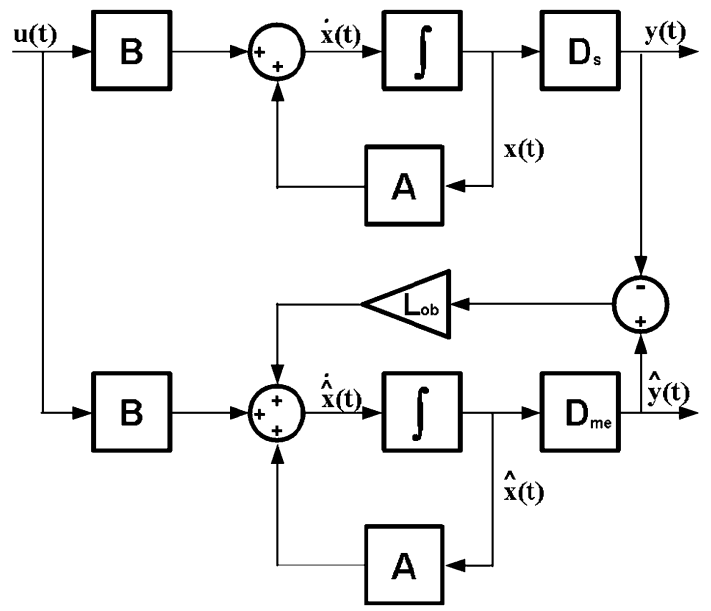

Fig. 3 General diagram of the state observer where $[Q]$ is a symmetric positive semi-definite state weighting matrix, and $[R]$ is a symmetric positive definite control effort weighting, which must meet the following properties:

$\left\{x^{T}\right\}[Q]\{x\} \geq 0, \quad \forall x \neq 0$

$\left\{u^{T}\right\}[R]\{u\} \geq 0, \quad \forall u \neq 0$

The differential of this work is that instead of using simulated data to minimize error, the data obtained experimentally by the sensors will be used, as represented in Fig. 4 .

In Eq. (3), the vector $\{u(t)\}$ is characterized by the rotor unbalance forces which are related to the unbalanced mass, $m_{o}$, which rotates with an angular velocity, $\Omega$, and is located at a distance from the center of rotation, $e$, as given by Eq. (10) [1]:

$\left[\begin{array}{l}f_{v}(t) \\ f_{w}(t)\end{array}\right]=m_{o} e \Omega^{2}\left[\begin{array}{c}\sin (\Omega t) \\ \cos (\Omega t)\end{array}\right]$

However, in many situations, the force responsible for excitation of the system is unknown. Thus, it is proposed to use a force identification method using orthogonal functions, Chebyshev polynomials specifically, to verify its effectiveness when compared to the real power system excitation.

The $\{x(t)\}$ and $\{f(t)\}$ signals are expanded into Chebyshev polynomials with an " $r$ " number, according to Eq. (11) [6].

$g(t) \cong \sum_{n=0}^{r-1} g_{n} \varphi_{n}(t)$

where the Chebyshev polynomials, for $n \geq 1$, are given by [11]:

$\left\{\varphi_{i+1}(t)\right\}=2\left(\frac{2 t}{t_{f}}-1\right)\left\{\varphi_{i}(t)-\varphi_{i-1}(t)\right\}$

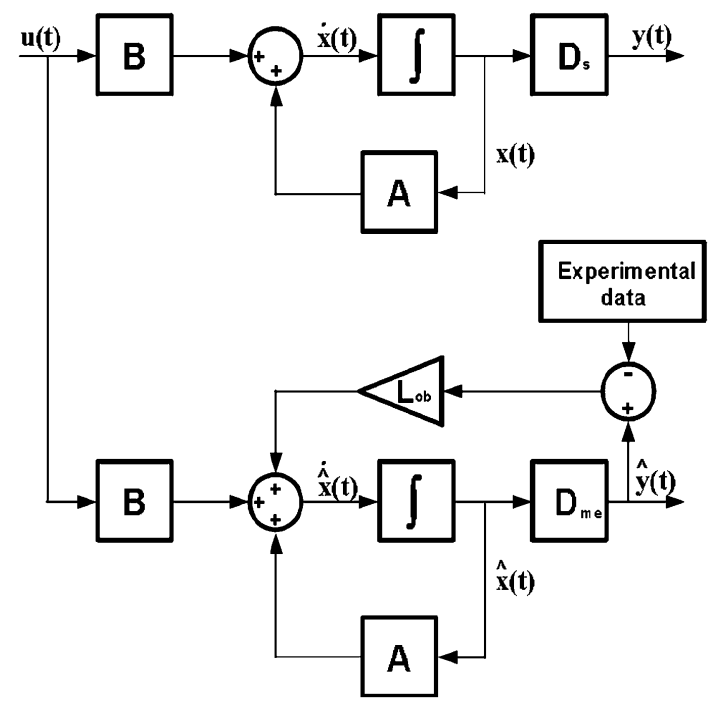

Fig. 4 Diagram of the state observer using experimental data 
with $\varphi_{0}(t)=1$ and $\varphi_{1}(t)=\frac{2 t}{t_{f}}-1$.

The $\{x(t)\}$ and $\{f(t)\}$ signals, as acquired, are expanded into series of orthogonal functions truncated, as shown by Eqs. (13) and (14).

$\{x(t)\}=[X]\{\varphi(t)\}$

$\{f(t)\}=[F]\{\varphi(t)\}$

where $[X]$ and $[F]$ are the displacement and force coefficients to Chebyshev polynomials $[6,8]$.

Evaluating the double integral of Eq. (2), results in Eq. (15) [6].

$$
\begin{array}{r}
(\{x(t)\}-\{x(0)\}-\{\dot{x}(0)\} t)+ \\
{[C]\left(\int_{0}^{t}\{x(\tau)\} d \tau-\{x(0)\}\right)+}
\end{array}
$$

$[K] \int_{0}^{t} \int_{0}^{t}\{x(\tau)\} d \tau^{2}=\int_{0}^{t} \int_{0}^{t}\{f(\tau)\} d \tau^{2}$

where $\tau$ is used as a variable in the integration of $[0, t]$.

Substituting Eqs. (13) and (14) into Eq. (15) yields Eq. (16):

$$
\begin{array}{r}
([X]\{\varphi(t)\}-\{x(0)\}-\{\dot{x}(0)\} t)+ \\
{[C]\left(\int_{0}^{t}[X]\{\varphi(\tau)\} \mathrm{d} \tau-\{x(0)\} t\right)+} \\
\left(\int_{0}^{t} \int_{0}^{t}[X]\{\varphi(\tau)\} \mathrm{d} \tau^{2}\right)=\int_{0}^{t} \int_{0}^{t}[F]\{\varphi(\tau)\} \mathrm{d} \tau^{2}
\end{array}
$$

A finite set of orthonormal functions have a particular property in relation to their successive integration in the interval $\left[0, T_{p}\right]$, according to Eq. (17) $[6,8]$.

$$
\int_{0}^{t} \underbrace{\ldots}_{\mathrm{n} \text { times }} \int_{0}^{t}\{\varphi(\tau)\}(\mathrm{d} \tau) \cong[P]^{n}\{\varphi(t)\}
$$

where $n$ is the number of times the function has been integrated and $[P]$ is a square matrix with constant elements, called the operational matrix of integration, related to Chebyshev polynomials [12].

Substituting Eq. (17) in Eq. (16) it is possible to obtain the Eq. (18) [6, 8, 12]:

$$
[F]=[P]^{-2}\left[\begin{array}{c}
{[M]} \\
-[M] x(0) \\
-[M] \dot{x}(0)-[C] \dot{x}(0) \\
{[C]} \\
{[K]}
\end{array}\right]^{T}\left[\begin{array}{c}
{[X]} \\
\{e\}^{T} \\
\{e\}^{T}[P] \\
{[X][P]} \\
{[X][P]^{2}}
\end{array}\right]
$$

where $\{e\}^{T}=\left\{\begin{array}{lll}1 & 0 & \ldots 0\end{array}\right\}$.

Finally, replacing the expanded terms of force, Eq. (18), and the Chebyshev polynomial terms, Eq. (12), it is possible to reconstruct the force of Eq. (14).

As can be seen in Eq. (10), the unbalance force is dependent on the angular velocity of the rotor. One way of avoiding this dependency is to use the moment of unbalance, which is the relationship between the unbalanced mass and its distance from the center of rotation, instead of the unbalance force. As the moment of unbalance is the same for both directions, it was chosen to identify it to the $x$ direction, as shown in Eq. (19).

$m_{o} e \sin (\Omega t)=\frac{f_{v}(t)}{\Omega^{2}}$

\section{Experimental validation}

The system consists of a circular shaft of steel in 1020, two ball bearings, a disk containing twelve holes around the center and a gear, as shown in Fig. 5.

The rotor was discretized into eleven finite elements, as shown in the rotor side view with the respective nodes in the Fig. 6.

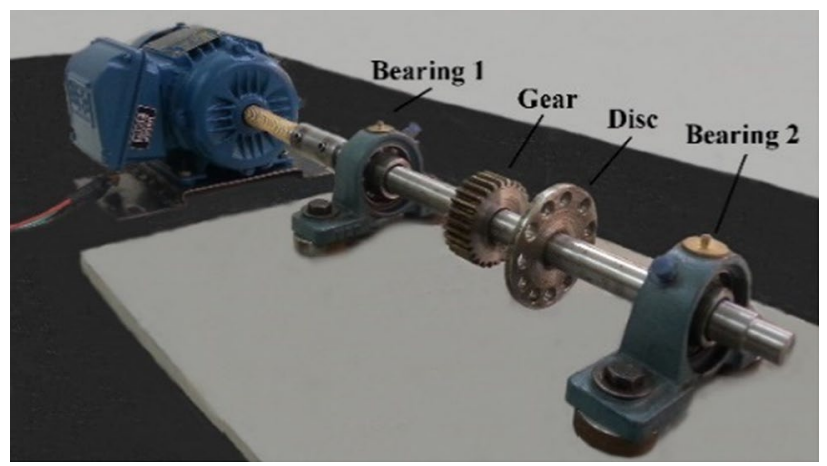

Fig. 5 Rotor test bench

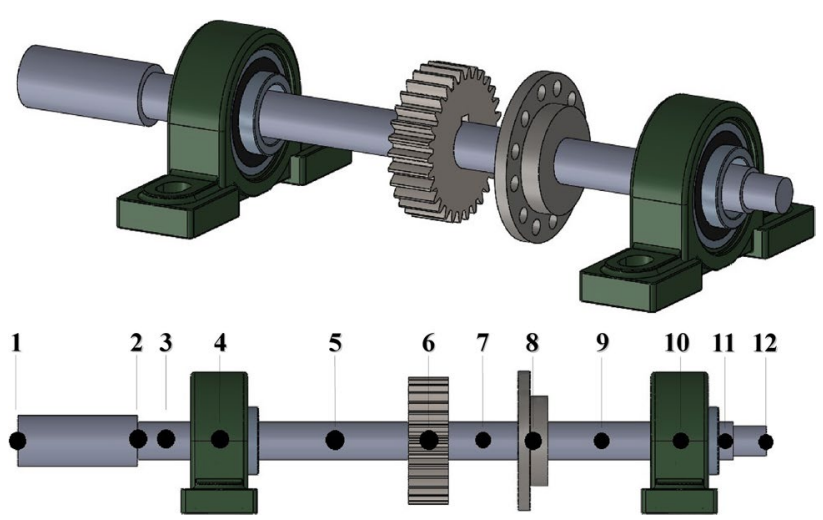

Fig. 6 Rotor discretization in finite element 
Table 2 Properties of components of the rotor

\begin{tabular}{lllc}
\hline & Disk & Gear & Bearings \\
\hline Mass $(\mathrm{kg})$ & 0.2394 & 0.2544 & 0.7 \\
$\begin{array}{c}\text { Moment of inertia in } x \\
\text { direction }\left(\mathrm{kg} \mathrm{m}^{2}\right)\end{array}$ & $64.6 \times 10^{-6}$ & $54.1 \times 10^{-6}$ & - \\
$\begin{array}{c}\text { Moment of inertia in } y \\
\text { direction }\left(\mathrm{kg} \mathrm{m}^{2}\right)\end{array}$ & $64.6 \times 10^{-6}$ & $54.4 \times 10^{-6}$ & - \\
$\begin{array}{c}\text { Moment of inertia in } z \\
\text { direction }\left(\mathrm{kg} \mathrm{m}^{2}\right)\end{array}$ & $120.8 \times 10^{-6}$ & $91.6 \times 10^{-6}$ & - \\
\hline
\end{tabular}

Table 3 Dimension of each element

\begin{tabular}{lll}
\hline Element number & Length $(\mathrm{mm})$ & Diameter $(\mathrm{mm})$ \\
\hline 1 & 60.00 & 25.56 \\
$2-3$ & 20.00 & 19.00 \\
$4-5$ & 52.50 & 19.00 \\
$6-7$ & 24.00 & 19.00 \\
$8-9$ & 39.00 & 19.00 \\
10 & 27.00 & 19.00 \\
11 & 16.00 & 15.00 \\
\hline
\end{tabular}

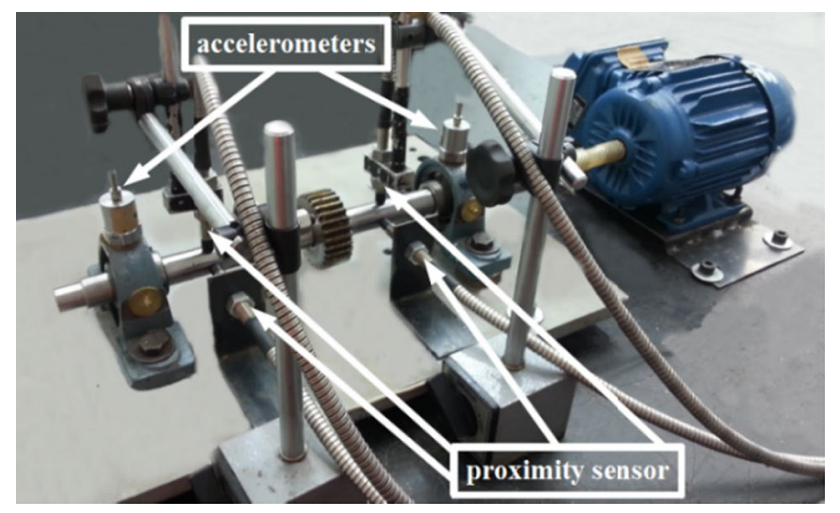

Fig. 7 Rotor bench with instrumentation

Table 2 shows the properties of the bearing, gear and disk and Table 3 shows the parameters for the rotor discretization.

The experimental bench measurements were obtained by means of sensors installed on the bearings and at the 5th and 9 th nodes of the shaft.

The displacement of the shaft was monitored by proximity sensors and the vibrations of the bearing by accelerometers, as shown in Fig. 7.

\section{Simulated and experimental results}

At first, the rotor balancing was performed on a plane, after which a mass was added in the disk, as shown in Fig. 8.

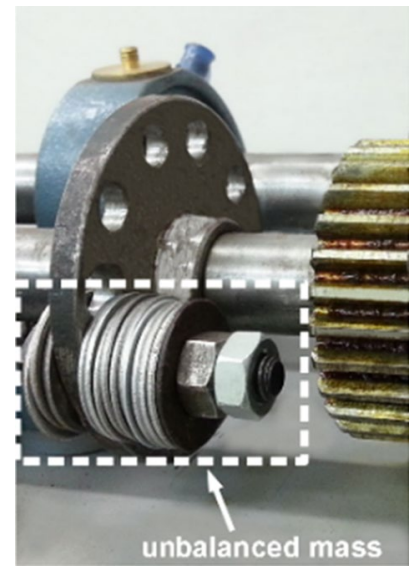

Fig. 8 Unbalance mass in the disk

The experimental data were filtered by a bandpass filter of 4 th order. The cutoff frequencies used were $\pm 10 \%$ of the rotation. The phase of the sensor was not used but the signals were windowed so they start at time zero with zero amplitude and end in an integer number of cycles. Figure 9 shows the experimental signal of the bearingsblue and red curves - and of the fifth and ninth nodesgreen and acqua curves - in $\mathrm{z}$ direction, before and after of filtered and windowed.

The data, after being processed, was inserted into the objective function and the genetic algorithm generated a vector solution, which was used as initial estimate for the quasi-Newton method. Finally, the solutions obtained are shown in Table 4.

Using the displacement data reconstructed by the observer, it was possible to apply the method of identification of the forces by Chebyshev polynomials, simulating rotation for two speeds, 1600 and $2000 \mathrm{rpm}$.

Using the Eq. (19), the unbalance moment calculated was close to $1.74 \times 10^{-4} \mathrm{~kg} \mathrm{~m}$, as can be seen in Figs. 10, 11 .

\section{Conclusions}

This work in this paper was aimed at implementing computational algorithms to estimate the stiffness and damping of bearings and to identify the unbalance forces acting on a rotor.

A hybrid metaheuristic algorithm was developed combining a genetic algorithm to estimate the unknown parameters of stiffness and damping of the bearings without the need to provide an initial solution, and then, use the solution found in the quasi-Newton method optimization.

The developed algorithms were tested with numerical simulations and, subsequently, the experimental data were used so that the algorithm estimated the parameters and the 


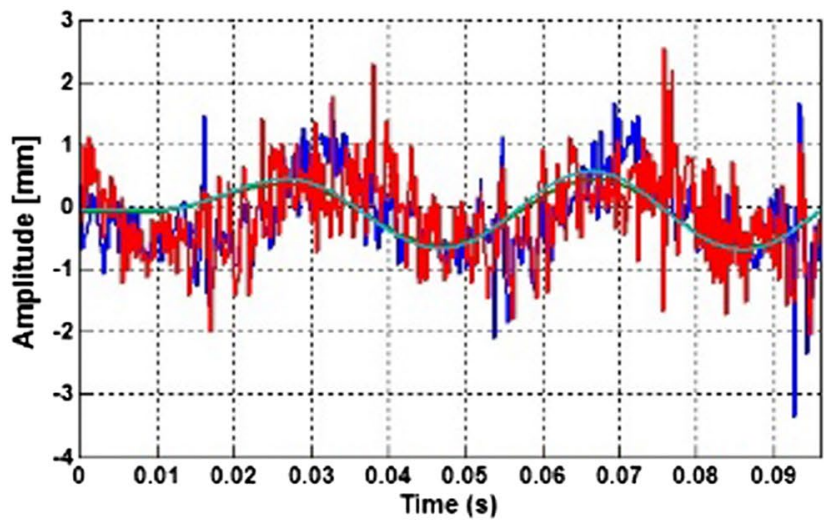

(a)

Fig. 9 a Original experimental signal. b Windowed experimental signal

Table 4 Bearing parameters

\begin{tabular}{lll}
\hline & $Z$ direction & $X$ direction \\
\hline Stiffness of the bearing 1 $(\mathrm{kN} / \mathrm{m})$ & 646 & 637 \\
Stiffness of the bearing 2(kN/m) & 839 & 864 \\
Damping of the bearing 1 (Ns/m) & 449 & 658 \\
Damping of the bearing 2 (Ns/m) & 469 & 577 \\
\hline
\end{tabular}

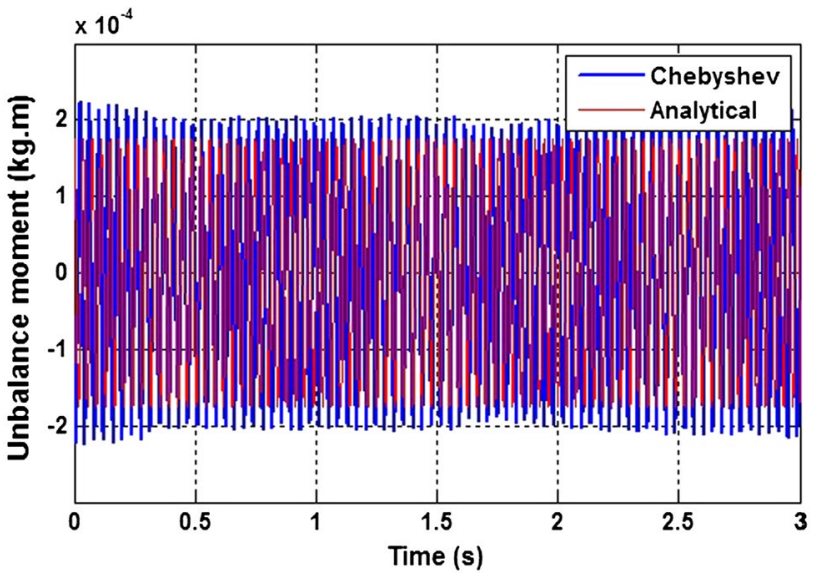

Fig. 10 Identification of the unbalance moment from $1600 \mathrm{rpm}$

state observer reconstructed the displacement and velocity signals. To verify the robustness of the parameters, experimental data for rotations of 1600 and $2000 \mathrm{rpm}$ were collected, changing, with it, the unbalance force of the system.

Finally, with all the states of the system and using Chebyshev polynomials, the unbalance forces were identified. Using Eq. (19), it was possible to calculate the unbalance moment of the system.

Estimation of stiffness and damping parameters through the hybrid metaheuristic method presented here was promising.

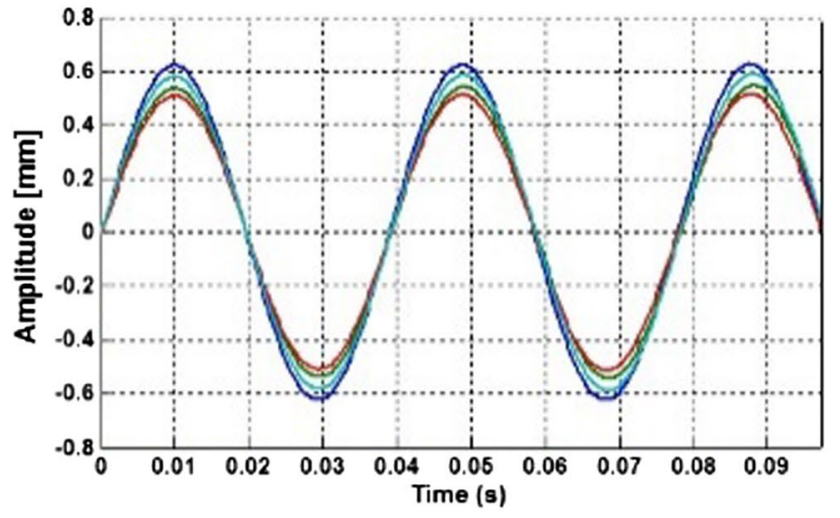

(b)

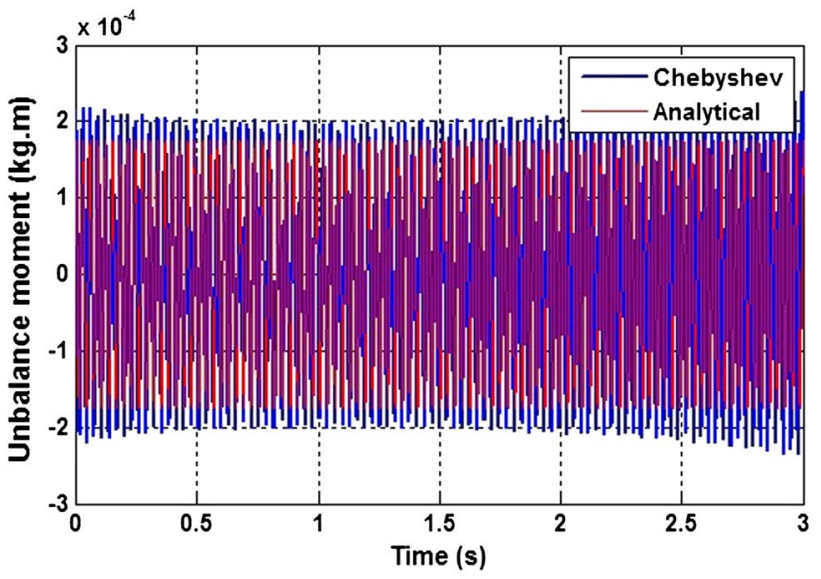

Fig. 11 Identification of the unbalance moment from $2000 \mathrm{rpm}$

They enabled the reconstruction of other states by the state observer and also the identification of forces with success.

Acknowledgments The authors thank INCT and CAPES for the financial support and the Department of Engineering Mechanics of Ilha Solteira for technical support.

\section{References}

1. Castro HF, Cavalca KL, Camargo LWF, Bachschmid N (2010) Identification of unbalance forces by metaheuristic search algorithms. Mech Syst Signal Process 24:1785-1798

2. Ford JA, Saadallah AF (1987) On the construction of minimization methods of quasi-Newton type. J Comput Appl Math 20:239-246

3. Lalanne M, Ferraris G (1997) Rotor dynamics prediction in engineering. 2.ed. John Wiley and Sons, 254p

4. Luenberger DG (1964) Observing the state of a linear system. IEEE Transactions on Military Ele. Piscataway 8:74-80

5. Martínez JM (2000) Practical quasi-Newton methods for solving nonlinear systems. J Comput Appl Math 124:97-121 
6. Melo GP, Morais TS (2007) Fault detection using state observers with unknown input, identified by orthogonal functions and PI observers. Solid Mech Struct 1:341-356

7. Mohamed $\mathrm{G}$ et al (2012) Optimal vibration control for structuralacoustic coupling system. J Vib Control 19:14-29

8. Pacheco RP, Júnior VS (2003) On the identification of non-linear mechanical systems using orthogonal functions. Int J Non-linear Mech 39:1147-1159

9. Shanno DF (1970) Conditioning of quasi-Newton methods for function minimization. Math Comput 24:647-656
10. Simões RC, Júnior VS (2003) Optimization techniques for fault identification in rotor dymanics. In: 17th International Congress of Mechanical Engineering, São Paulo, Brazil

11. Xiaopeng J, Long G, Zhiquan W (2008) Identification of one class of distributed parameter systems based on Chebyshev polynomials. In: 27th Chinese Control Conference, Kunming, China

12. Zhang $J$ et al (2015) Fault diagnosis approach for rotating machinery based on dynamic model and computational intelligence. Measurement 59:73-87 